\title{
Influence of microdamage structure on fatigue resistance of metal functional material
}

\author{
Maria Bartolomey \\ Department of Computational Mathematics and Mechanics \\ Perm National Research Polytechnic University \\ Perm, Russia \\ mbartolomey@mail.ru \\ Dmitriy Boyarshinov \\ Department of Computational Mathematics and Mechanics \\ Perm National Research Polytechnic University \\ Perm, Russia
}

\author{
Sergei Batin \\ Department of Computational Mathematics and Mechanics \\ Perm National Research Polytechnic University \\ Perm, Russia \\ Mikhail Gitman \\ Department of Computational Mathematics and Mechanics \\ Perm National Research Polytechnic University \\ Perm, Russia \\ gmb@pstu.ru
}

\author{
Vladimir Oborin \\ Laboratory of physical foundation of strength \\ Institute of Continuous Media Mechanics of Ural branch of RAS \\ Perm, Russia \\ oborin@icmm.ru
}

\begin{abstract}
The role of the collective behavior of defect ensembles at the crack tip and the laws of fatigue crack propagation in aluminum-magnesium alloy AMg6 have been studied under conditions of symmetric tension-compression gigacycle loading at $20 \mathrm{kHz}$ using ultrasonic fatigue testing machine Shimadzu USF2000. 3D New View 5010 interferometer profiler high resolution data of defect induced roughness in the crack process zone under fatigue crack path revealed the existence of two characteristic scales: the scale of the process zone and the correlation length that is the scale when the correlated behavior of defect induced roughness has started. A mathematical model allowing the assessment of fatigue resistance of functional metal material on the basis of the analysis of loading parameters and the resulting fracture surface is developed. Analyzing the image by means of the wavelet-analysis, the basis parameters of a destruction surface are allocated. Based on the analysis of the destruction surface characteristics and the corresponding loading parameters, the fatigue life of the functional material is evaluated. For the solution of the problem, the elements of the fuzzy sets theory are used.
\end{abstract}

Keywords-functional material; microstructure; morphology; microdamage; fatigue resistance

\section{INTRODUCTION}

In recent years, the role of metal alloys as functional materials constantly increases in the modern industry. Such materials have the set operating characteristics (durability, conductivity, thermal stability, etc.) formed due to use of new manufacturing technologies and/or special further thermomechanical treatment [1-3], and, as a result, the organized microstructure with particular grain-phase structure and microdamage. At the same time, necessity of development of new tools of the analysis of difficult microstructures and forecasting of strength properties at the microscale levels increases.

The list of the functional materials applied in mechanical engineering and instrument engineering is large, and it is possible to classify them by various signs. Most of them like irons, alloys on the basis of copper and light metals, are universal. They have numerous advantages and are used in various components and constructions.

Together with universal a functional materials of a certain functional purpose are applied: high-temperature-resistant materials, materials with high elastic properties, wearproof, corrosion resisting and etc.

It is possible to classify functional materials by the properties defining the choice of material for specific components of a construction. Each class of materials is estimated by the criterion providing their working capacity and materials are chosen taking into account their properties: mechanical, physical, technological, operational, etc. For structural materials, the following properties are considered: durability, plasticity, viscosity, elasticity, hardness, brittleness.

Assessment of strength properties of metal functional materials depending on types of microdamage is of considerable interest. Many scientists are interested in a study of microdamages of the functional materials, see e.g. development of damages with use of numerical algorithms [4], 
or experimentally [5-6]. A number of works are devoted to a research of microdamages that have arisen due to thermal treatment of materials [7], the works directed to studying of the microdamages that have arisen during the working process also existed [3,8].

During operation, most parts and machines are subject to cyclic loads, thus the fatigue failure can be considered the most common type of operational failure. At fatigue failure there is a development of areas of localization of damages in the field of microcracks which parameters significantly influence to the fatigue durability of the material. Geometrical damages (for example cuts, chips) of metal functional materials and their microstructure also have significant influence on the fatigue resistance of a construction [9-10]. The problem of fatigue failure of metals and alloys attracts interest as often a harbinger of brittle fracture of material is emergence of a fatigue crack that leads to decrease in the bearing capacity [11]. Appearance of new functional materials demands new approaches to assessment of their structure and strength properties at various modes of operation. Thus, development of new methods of the analysis of fatigue properties of materials and forecasting of strength characteristics is the issue of the day.

The main focus of this paper is the development of the methodology, allowing the assessment of fatigue resistance of functional metal material on the basis of the analysis of loading parameters and the resulting fracture surface. A novelty of the developed estimation technique is the application of the fuzzy sets theory in establishing multiple relations between a given complex of strength characteristics and parameters of microdamage, obtained from fracture surface images in the analysed material.

\section{DEFINITION OF THE EXPERIMENT}

In this paper, the study of the growth kinetics of fatigue cracks in aluminum and magnesium alloy AMg6 under conditions of high- and gigacyclic fatigue is presented, in materials find new regularities of destruction connected with qualitative changing in mechanisms of rising and development of fatigue cracks is conducted [12]. The samples were loaded on the ultrasonic fatigue testing machine Shimadzu USF2000. The ultrasonic testing machine allows to test samples on the basis of $10^{9}-10^{10}$ cycles with an amplitude from 1 and to several tens microns with a frequency of $20 \mathrm{kHz}$, it reduces test time to several days in contrast to classical fatigue installations in which such number of cycles is reached for years of tests. All experiments were conducted by Laboratory of Physical Foundation of Strength ICMM UrB RAS.

Chemical composition and quasi-static tensile characteristics of AMg6 alloy are shown in Tables I and II.

TABLE I. CHEMICAL COMPOSITION OF AMG6 (IN WEIGHT \%)

\begin{tabular}{|c|c|c|c|c|c|c|c|c|}
\hline $\mathbf{A l}$ & $\mathbf{C u}$ & $\mathbf{M g}$ & $\mathbf{M n}$ & $\mathbf{S i}$ & $\mathbf{F e}$ & $\mathbf{Z n}$ & $\mathbf{B e}$ & $\mathbf{T i}$ \\
\hline $\begin{array}{c}91.1- \\
93.68\end{array}$ & 0.10 & $\begin{array}{c}5.8- \\
6.8\end{array}$ & $\begin{array}{c}0.5- \\
0.8\end{array}$ & 0.4 & 0.4 & 0.20 & $\begin{array}{c}0.0002- \\
0.005\end{array}$ & $\begin{array}{c}0.02- \\
0.1\end{array}$ \\
\hline
\end{tabular}

TABLE II. QUASI-STATIC TENSILE CHARACTERISTICS OF AMG6

\begin{tabular}{|c|c|c|c|}
\hline $\begin{array}{c}\text { Elastic modulus } \\
\text { (GPa) }\end{array}$ & $\begin{array}{c}\text { Yield } \\
\text { stress } \\
\text { (MPa) }\end{array}$ & $\begin{array}{c}\text { Tensile } \\
\text { strength } \\
\text { (Mpa) }\end{array}$ & $\begin{array}{c}\text { Ultimate } \\
\text { elongation (\%) }\end{array}$ \\
\hline 71 & 180 & 355 & 25 \\
\hline
\end{tabular}

The samples (Fig. 1) of aluminum-magnesium alloy AMg6 were tested under symmetric tension-compression fatigue loading at $20 \mathrm{kHz}$ (mode of gigacyclic loading) at stress level $158,150,140,138,130,120 \mathrm{MPa}$ on the ultrasonic fatigue testing machine (Shimadzu USF2000). The testing machine consists of the following main parts: the generator transforming the frequency of $50 \mathrm{~Hz}$ to an ultrasonic electric sine waveform with a frequency of $20 \mathrm{kHz}$; the piezoelectric transducer generating longitudinal ultrasonic waves in mechanical impact with a frequency of $20 \mathrm{kHz}$; the ultrasonic waveguide creating the maximum amplitude of mechanical stress in a working (mid-section) part of a sample. Deviation of the frequency by $0.5 \mathrm{kHz}$ was associated with the damage critical stage and considered as failure precursor related to the initiation of a crack with characteristic size of $\sim 2 \mathrm{~mm}$.

In total 8 samples have been investigated.

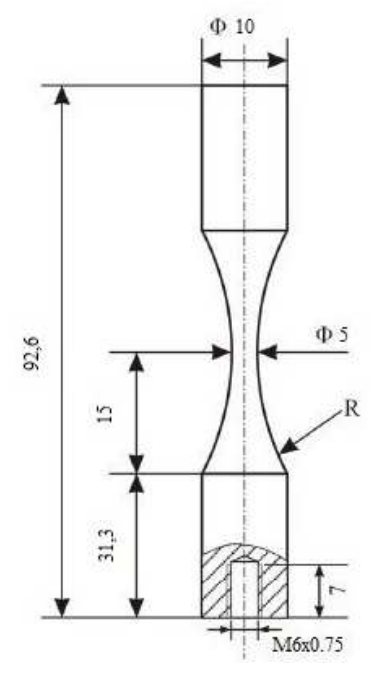

Fig. 1. Sample geometry (sizes are given in millimeters).

The roughness of destruction surfaces was measured by the high-resolution New-View 5010 interferometer-profiler (providing x2000 magnification) and then was analyzed using the assumption concerning fractal geometry of fracture surface profile associated with correlated behavior of multiscale defect structures on the scale of process zone $L_{p z}$ that preceded to the crack growth.

The scanning areas were located in zone 2 (Fig. 2.a). About 10 one-dimensional "cuts" were analyzed within each "window", providing representative data of the relief structure induced by damages with the vertical permission $\sim 0.1 \mathrm{~nm}$ and horizontal permission $\sim 0.1 \mathrm{mkm}$. Table III shows the values of stress levels and fatigue life for the samples under study. 


\section{a)}
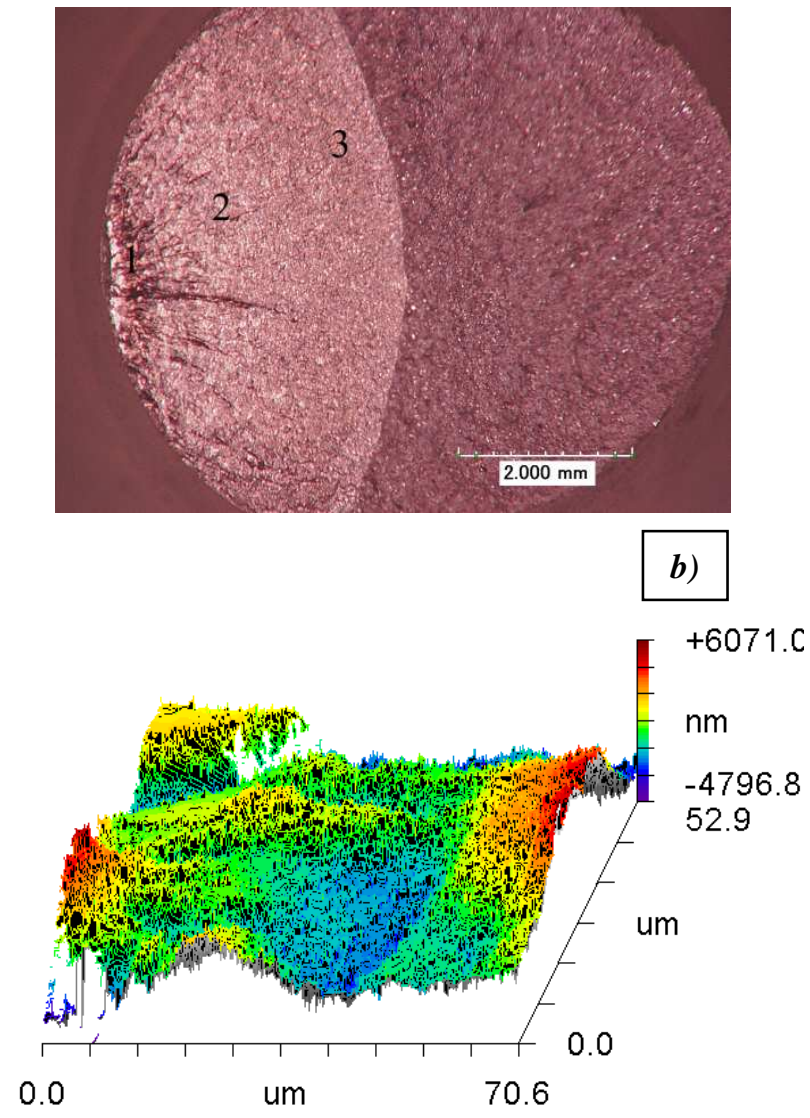

Fig. 2. Typical relief of a surface of an area of fatigue failure (a) optical image, (b) 3D image of a surface of area 1

TABLE III. THE VALUES OF THE HURST EXPONENT AND THE CRITICAL SCALE AT VARIOUS LEVELS OF FATIGUE LIFE

\begin{tabular}{|c|c|c|c|c|}
\hline № & $\underset{\mathrm{MPa}}{\sigma}$ & $\begin{array}{c}l_{s c}, \\
\mathbf{m k m}\end{array}$ & $L_{p z}, \mathbf{m k m}$ & $H$, Hurst exponent \\
\hline 1 & 158 & 1 & 20.3 & 0.63 \\
\hline 2 & 150 & 1.1 & 12.3 & 0.49 \\
\hline 3 & 140 & 2.0 & 18.2 & 0.66 \\
\hline 4 & 138 & 1.4 & 17.6 & 0.48 \\
\hline 5 & 130 & 4.3 & 36.3 & 0.79 \\
\hline 6 & 120 & 2.8 & 20.7 & 0.63 \\
\hline 7 & 130 & 1.6 & 19.7 & 0.58 \\
\hline 8 & 130 & 1.1 & 15.2 & 0.72 \\
\hline № & \multicolumn{2}{|c|}{$\begin{array}{c}\Delta N, \text { cycles } \\
\text { (fatigue life) }\end{array}$} & \multicolumn{2}{|c|}{$\underset{\text { (fatigue life) }}{\lg \Delta N, \text { cycles }}$} \\
\hline 1 & \multicolumn{2}{|c|}{$8.5 \cdot 10^{+5}$} & \multicolumn{2}{|r|}{5.9294} \\
\hline 2 & \multicolumn{2}{|c|}{$5.98 \cdot 10^{+5}$} & \multicolumn{2}{|r|}{5.7767} \\
\hline 3 & \multicolumn{2}{|c|}{$2.72 \cdot 10^{+8}$} & \multicolumn{2}{|r|}{8.4346} \\
\hline 4 & \multicolumn{2}{|c|}{$4.79 \cdot 10^{+6}$} & \multicolumn{2}{|r|}{6.6803} \\
\hline 5 & \multicolumn{2}{|c|}{$8.6 \cdot 10^{+5}$} & \multicolumn{2}{|r|}{5.9345} \\
\hline 6 & \multicolumn{2}{|c|}{$2.52 \cdot 10^{+7}$} & \multicolumn{2}{|r|}{7.4014} \\
\hline 7 & \multicolumn{2}{|c|}{$1.50 \cdot 10^{+9}$} & \multicolumn{2}{|r|}{9.1761} \\
\hline 8 & \multicolumn{2}{|c|}{$8.83 \cdot 10^{+8}$} & \multicolumn{2}{|r|}{8.9460} \\
\hline
\end{tabular}

A minimum (critical) scale $l_{s c}$ that corresponds to beginning of multiscale long-range correlation in defect ensembles is determined by the computing of the Hurst exponent [13]. The function $K(d)$ is calculated for onedimensional profiles of fracture surface relief according to the formula:

$$
K(d)=\left\langle(z(x+d)-z(x))^{2}\right\rangle_{x}^{1 / 2} \propto d^{H}
$$

where $K(d)$ is the averaged difference between the values of surface relief heights $z(x+d)$ and $z(x)$ in the window of size $d$, and $H$ is the Hurst exponent (surface roughness index).

Representation of the function $K(d)$ in logarithmic coordinates allowed one to evaluate the lower boundary of the scaling range $l_{s c}$, and the value of upper boundary considering one as the characteristic scale of the process zone $L_{p z}$, i.e. the area of correlated behavior of multiscale defect structures (Fig. 3.).

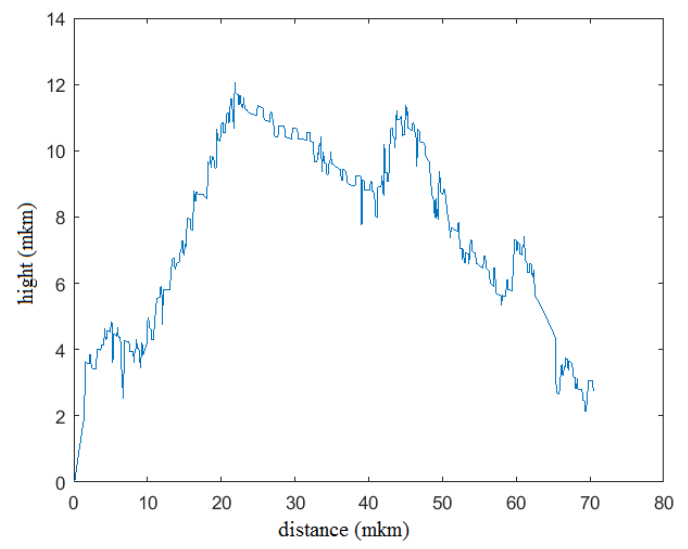

Fig. 3. Characteristic one-dimensional profiles

The values of the Hurst exponent $H$ and the scales $L_{p z}$ and $l_{s c}$ for different loading conditions are given in Table III.

The data revealed slight variation of the roughness exponent for different number of cycles.

\section{MATHEMATICAL MODEL}

Let us assume that the digital image of a destruction surface of the studied functional material and the loading parameters corresponding to this surface are set. Analyzing the image by means of the wavelet-analysis [14], it is possible to allocate the basis parameters of a destrustion surface to which it is possible to relate: $l_{s c}$ - correlation scale in the ensemble of defects; $L_{p z}$ - the scale connected with a process zone and $H-$ Hurst exponents [15]. The number of the chosen parameters is indicated as a variable $k$.

Based on the analysis of the destruction surface characteristics and the corresponding loading parameters, it is required to evaluate the fatigue life of the functional material.

Let us assume that the number of the parameters characterizing fatigue resistance of the functional material is equal to $r$. It is required to develop the general technique, 
allowing one to establish the relations between fatigue resistance of the functional material on the one hand, and loading parameters and characteristics of the destruction surface on the other hand, on the available limited set of experimental data.

For the solution of the problem the elements of the fuzzy sets theory are used [16]. Fuzzy relations Si between loading parameters and destruction surface and parameters of fatigue resistance are formulated. Similar approach was used for creation of the relation between parameters of grain-phase structure of the material and its operating characteristics, in particular, by strength properties [17].

Let us assume that the number of the available experiments is equal to $l$. Then:

$$
S^{i}=A^{i} \times B^{i}, i=1, \ldots, l,
$$

where $A^{i}$ - fuzzy set containing loading parameters and received surface of destruction for $i$-th experiment; $B^{i}-$ fuzzy set containing characteristics of fatigue resistance for $i$ th experiment; $X$ - sign of Cartesian product of fuzzy sets [16].

Let us note that (1) determines by itself the fuzzy relation which is represented in the form of a matrix where $m n$-th member of this matrix $\mu_{m n}$ defined for the element $\left(a_{m}, b_{n}\right)$ is determined by rules of vector product for fuzzy sets $[16] ; m=1, \ldots, d ; \quad n=1, \ldots, w$.

It should be marked that total quantity of couples of elements in the fuzzy set $A^{i}$ is equal to value $d$ and in the fuzzy set $B^{i}$ is equal to value $w$ which are defined as follows:

$$
d=\sum_{i=1}^{k} p_{l} ; w=\sum_{i=1}^{r} t_{l}
$$

Thus for each $i$-th experiment $(i=1, \ldots, l)$ the relation of communication $S^{i}$ between fuzzy sets $A^{i}$ and $B^{i}$ is defined.

Connection between arbitrary (testing) set $A^{\text {test }}$ describing loading parameters and received surface of destruction of the functional material and the set $B^{\text {test }}$ induced by him describing characteristics of fatigue resistance of the material can be defined as follows [16]:

$$
S=\bigcup_{i=1}^{l} S^{i}
$$

At the same time the induced set $B^{\text {test }}$ is defined by relations:

$$
B^{\text {test }}=A^{\text {test }} \circ S \text {, }
$$

where "०" denotes maximine product (maximine product is defined as the usual product of matrixes where instead of operation of product $\min$ is entered, and instead of composition operation - $\max$ ) [16].

It should be marked that $B^{\text {test }}$ will obtained as fuzzy set. If we are interested in $B^{\text {test }}$ in the form of scalar value (the fatigue resistance has to be presented as unambiguously certain scalar value), it is necessary to solve a problem of definition of the exact representative of the nonfuzzy number. It is possible to offer several ways of the solution of the problem considered, for example, in [17].

For assessment of accuracy of the prediction it is possible to use the following technique. Let the number of experiments is equal to $n$. For assessment of accuracy of calculations it is necessary to choose one of the available experiments for basic, i.e. the value of the studied characteristic for the experiment is marked as $R^{e x}$, and all remained experiments allow to determine the value $R^{\text {theor }}$ by the technique described above.

Now the error of the carried out calculations can be estimated on a function:

$$
\delta=\left|\frac{R^{\text {theor }}-R^{e x}}{R^{e x}}\right| \cdot 100 \%
$$

\section{CONSIDERATION OF THE RESUltS}

We demonstrate the presented methodology to estimate the fatigue life of a functional material and the accuracy of the prediction of the result.

As it was mentioned above together with Institute of Continuous Media Mechanics (ICMM UrB RAS) 8 experiments have been conducted (Table III). The 4th experiment was chosen as a reference experiment.

By results of 7 experiments (excluding the experiment №4), following the methodology described above, the characteristic of fatigue resistance was found to be 6.6803 (characteristics of fatigue resistance are presented in a logarithmic scale), it corresponds to $4.79 \cdot 10+6$ cycles before destruction. The relative error following (6) was estimated as $8.91 \%$. Let us note that to raise the accuracy of calculations it is necessary to increase the number of initial experiments.

\section{CONCLUSIONS}

In this work, a technique has been developed, allowing one to estimate the fatigue life of functional materials based on the loading parameters and the destruction surface analysis. The novelty of the offered technique is the application of the fuzzy set theory at formulation of multiple relations between loading parameters and the destruction surface of functional material on the one hand and characteristics of fatigue resistance from the other hand. The example of characteristics of fatigue resistance calculation and assessment of accuracy of expected calculations is given. 
The obtained results confirm the possibility of using the developed technique for complex assessment of fatigue resistance in loading parameters and destruction surface of the functional material. The results show that mentioned characteristic scales $L_{p z}$ and $l_{s c}$ play an important role in the list of variables for the kinetic equation fatigue of crack growth and can be used for the identification of phenomenological parameters (power index) providing self-similar law for the crack path [15].

It should be noted the practical importance of the presented research. Thus, for example, when investigating the causes of catastrophes (e.g. in aviation), it is possible to estimate the operational characteristics of the product under investigation based on the characteristics of the material destruction surface. If the parameters are higher than permissible, it demonstrates violation of time regulations of operation.

\section{Acknowledgment}

The reported study was funded by the Ministry of Education and Science of the Russian Federation (the unique identifier RFMEFI58617X0055) and by the EC Horizon 2020 is MSCA-RISE-2016 FRAMED Fracture across Scales and Materials, Processes and Disciplines.

\section{References}

[1] A.R. Shkolnikov, V.V. Ovchinnikov, N.V. Guschina, F.F. Mahinko, L.S Chemerinskaya, S.M. Mozharovskiy, V.A. Kozlovskih, L.I. Kaigorodova, " Change of dislocation structure and phase composition of alloy AmG6 at radiation by Ar ions with energy $40 \mathrm{keV}$, "Vestnik of Tomsk Polytechnic University, vol. 308, no. 7, pp. 58-64, 2005.

[2] D.Said Schicchi, A. Caggiano, T. Lübben, M. Hunkel, F. Hoffmann, "On the Mesoscale Fracture Initiation Criterion of Heterogeneous Steels During Quenching," Materials Performance and Characterization, vol. 6, no. 1, pp. 80-104, 2017. https://doi.org/10.1520/MPC20160098. ISSN 2165-3992.

[3] S.A. Loskutov, Yu.A. Bukin, Yu.D. Koryagin, "Research of metal microdamage of pipes of high temperature vapor lines from cr-mo-v steel of thermal power station at long operation", Vestnik of South-Ural State University. Series: Metallurgy, vol.15, no. 1, pp. 24-28, 2015.

[4] M. Takashi, T. Cristian, M. Daisuke and etc., "Mesoscale simulation of the early evolution of ductile fracture in dual-phase steels", international Journal of Plasticity, vol. 74, pp. 17-34, November 2015.
[5] S.A. Kumar, S.G.S. Raman, T.S.N.S. Narayanan, "Influence of surface mechanical attrition treatment duration on fatigue lives of Ti-6Al-4V", Transactions of the Indian Institute of Metals, vol. 67, pp. 137-141, February 2014.

[6] J. Yu, H. Zhang, D. Deng, Q. Liu, S. Hao, “Analysis of the relationship of crack arrest effects with fusion zone size by current detour and Joule heating", International Journal of Advanced Manufacturing Technology, vol. 87, pp. 1465-1474, November 2016.

[7] N. Wu, X. Zhang, S. Tu, X. Qian, Y. Wang, "Grian size effect on the initiation and propagation of small fatigue crack of GH4169 alloy at room temperature and $650^{\circ} \mathrm{C}$ ", Jixie Gongcheng Xuebao/Journal of Mechanical Engineering, vol. 52, pp. 66-75, October 2016.

[8] L. Bodelota, J. P. Escobedo-Diazb, C. P. Trujillob, D. T. Martinezb, and etc., "Microstructural changes and in-situ observation of localization in OFHC copper under dynamic loading", International Journal of Plasticity, vol. 74, pp. 58-74, November 2015.

[9] L.Signor, P.Villechaise, T.Ghidossi, E.Lacoste, M.Gueguen, S.Courtin, "Influence of local crystallographic configuration on microcrack initiation in fatigued $316 \mathrm{LN}$ stainless steel: Experiments and crystal plasticity finite elements simulations", Materials Science and Engineering A, vol. 649, pp. 239-249, January 2016.

[10] R. Guerchais , N .Saintier , F. Morel, C. Robert, "Micromechanical investigation of the influence of defects in high cycle fatigue", International Journal of Fatigue, vol. 67, pp. 159-172, October 2014.

[11] V.G. Pachurin, V.V. Galkin, G.V. Pachurin, A.A. Derbenev, "Assessment of a structural state influence on cyclic durability of the deformed materials", Transactions of NNSTU n.a. R.E. Alekseyev, no. 4 (111), pp. 177-185, 2015.

[12] P. Paris, D. Lados, H. Tad, "Reflections on identifying the real $\Delta$ Keffective in the threshold region and beyond", Engineering Fracture Mechanics, vol. 75, pp. 299-3052, 2008.

[13] E. Bouchaud, "Scaling properties of cracks", J. Phys. Condens. Matter, vol. 9, pp. 4319-4344, 1997.

[14] I. Daubechies, I. Guskov, P. Schryoder, W. Sweldens, "Wavelets on Irregular Point Sets”, Phil.Trans.: Math., Physical,Engng.Sci., vol. 357, pp. 2397-2413, 1999.

[15] V.A. Oborin, M.V. Bannikov, Y.V. Bayandin, M.A. Sokovikov, D.A. Bilalov, O.B Naimark, "Fractal analysis of fracture surface of aluminum alloy AMg6 under fatigue and dynamic loading", PNRPU Mechanics Bulletin, no. 2, pp.116-126, 2015.

[16] L. Zadeh, "From computing with numbers to computing with words from manipulation of measurements to manipulation of perceptions", International Journal of Applied Math and Computer Science, vol. 12, no. 3, pp. 307-324, 2002.

[17] I.M. Gitman, A.V. Klyuev, M.B. Gitman, V.Yu. Stolbov, "Multi-scale approach for strength properties estimation in functional materials," ZAngewMathMech, in press. 